\title{
Soluble Epoxide Hydrolase Pharmacological Inhibition Decreases Alveolar Bone Loss by Modulating Host Inflammatory Response, RANK-Related Signaling, Endoplasmic Reticulum Stress, and Apoptosis ${ }^{\mathbb{1}}$
}

\author{
Carlos Antonio Trindade-da-Silva, ${ }^{1}$ Ahmed Bettaieb, ${ }^{1}$ Marcelo Henrique Napimoga, \\ Kin Sing Stephen Lee, Bora Inceoglu, Carlos Ueira-Vieira, Donald Bruun, \\ Sumanta Kumar Goswami, Fawaz G. Haj, and Bruce D. Hammock
}

Department of Entomology and Nematology and University of California, Davis Comprehensive Cancer Center (C.A.T.-S., K.S.S.L., B.I., S.K.G., B.D.H.), Nutrition Department (F.G.H.), and Department of Molecular Biosciences, School of Veterinary Medicine (D.B.), University of California, Davis, California; Institute of Genetics and Biochemistry, Federal University of Uberlândia, Uberlândia, Brazil (C.A.T.-d.-S., C.U.-V.); Laboratory of Immunology and Molecular Biology, São Leopoldo Mandic Institute and Research Center, Campinas, Brazil (M.H.N.); and Department of Nutrition, University of Tennessee-Knoxville, Knoxville, Tennessee (A.B.)

Received November 29, 2016; accepted March 24, 2017

\begin{abstract}
Epoxyeicosatrienoic acids (EETs), metabolites of arachidonic acid derived from the cytochrome P450 enzymes, are mainly metabolized by soluble epoxide hydrolase (sEH) to their corresponding diols. EETs but not their diols, have anti-inflammatory properties, and inhibition of sEH might provide protective effects against inflammatory bone loss. Thus, in the present study, we tested the selective sEH inhibitor, 1-trifluoromethoxyphenyl-3-(1-propionylpiperidin-4-yl) urea (TPPU), in a mouse model of periodontitis induced by infection with Aggregatibacter actinomycetemcomitans. Oral treatment of wild-type mice with TPPU and sEH knockout (KO) animals showed reduced bone loss induced by $A$. actinomycetemcomitans. This was
\end{abstract}

associated with decreased expression of key osteoclastogenic molecules, receptor activator of nuclear factor-кB/RANK ligand/osteoprotegerin, and the chemokine monocyte chemotactic protein 1 in the gingival tissue without affecting bacterial counts. In addition, downstream kinases p38 and c-Jun N-terminal kinase known to be activated in response to inflammatory signals were abrogated after TPPU treatment or in sEH KO mice. Moreover, endoplasmic reticulum stress was elevated in periodontal disease but was abrogated after TPPU treatment and in sEH knockout mice. Together, these results demonstrated that sEH pharmacological inhibition may be of therapeutic value in periodontitis.

\section{Introduction}

Periodontitis is a chronic inflammatory disease of the supporting tissues of the teeth with progressive attachment loss and bone destruction (Flemmig, 1999). Dysbiosis is thought to

This work was supported by National Institutes of Health National Institute on Environmental Health Sciences/Superfund Research Program [Grants R01ES002710, P42ES004699, ES025598-01A1, and R00ES024806] and Brazilian funding agencies São Paulo Research Foundation (FAPESP) and National Council for Scientific and Technological Development (CNPq). M.H.N. was supported by Grant 2015/23556-0 (FAPESP) and Grant 303555/2013-0 (CNPq); F.G.H. laboratory is supported by National Institutes of Health [Grants R01DK090492 and R01DK095359]; A.B. is supported by National Institutes of Health National Institute of Diabetes and Digestive and Kidney Diseases [Grant R00DK100736].

A.B., B.I., S.K.G., F.G.H., and B.D.H. are coinventors on patents related to soluble exposide hydrolase by the University of California; B.I. and B.D.H. are cofounders of Eicosis. The authors declare no competing financial interests.

${ }^{1}$ C.A.T.-d.-S. and A.B. contributed equally to this work.

https://doi.org/10.1124/jpet.116.238113.

S This article has supplemental material available at jpet.aspetjournals.org. be one of the major drivers of this condition. Imbalance in the periodontal microbiota, more specifically the relative abundance of individual species of bacteria, was hypothesized to affect the host-microbe interactions, ultimately leading to destructive inflammation and bone loss (Hajishengallis et al., 2012). However, more recent findings bring up the possibility that overgrowth of bacteria may be a resulting outcome rather than the cause of periodontitis. Thus, it is likely that the host response to bacteria that leads to the tissue changes noted in gingivitis and periodontitis is the root cause (Bartold and Van Dyke, 2013).

In chronic osteolytic inflammatory diseases such as periodontitis, the failure of endogenous resolution pathways seems to lead to tissue destruction, and, conversely, augmentation of these natural defensive mechanisms can be an effective approach to control these diseases (Van Dyke, 2011). Resolution of inflammation is now widely accepted as an active process, in which endogenous anti-inflammatory and proresolving

ABBREVIATIONS: ANOVA, analysis of variance; ARA, arachidonic acid; COX, cyclooxygenase; DHET, dihydroxy-eicosatrienoic acid; EET, epoxyeicosatrienoic acid; ER, endoplasmic reticulum; HETE, hydroxy-eicosatetraenoic; JNK, c-Jun N-terminal kinase; KO, knockout; LOX, lipoxygenase; MCP-1, monocyte chemotactic protein 1; OPG, osteoprotegerin; PD, periodontal disease; PEG, polyethylene glycol; RANK, receptor activator of nuclear factor-кB; RANKL, RANK ligand; sEH, soluble epoxide hydrolase; TPPU, trifluoromethoxyphenyl-3-(1-propionylpiperidine-4-yl) urea. 
mechanisms actively mediate wound healing and tissue regeneration as opposed to fibrosis and scarring (Levy et al., 2012; Ortega-Gómez et al., 2013). More recent studies provide supporting evidence for this concept, in which endogenous control of inflammation directly improves bone healing and regeneration and suppresses the flow of destructive inflammatory infiltrate into the tissue (Hasturk et al., 2007; Napimoga et al., 2012).

Arachidonic acid (ARA) is an omega-6 polyunsaturated fatty acid constituting the phospholipid domain of most cell membranes. It is released by phospholipases such as cytoplasmic phospholipase $\mathrm{A}_{2}$ and is metabolized into eicosanoids through three main routes, via prostaglandin-endoperoxide synthase/ cyclooxygenases (COX), lipoxygenases (LOX), and via the cytochrome P450s, resulting in prostanoids leukotrienes and hydroxy-eicosatetraenoic acids (HETE) and epoxyeicosatrienoic acids (EETs). The cytochrome P450 enzymes that use ARA as a substrate mainly produce EETs and the $\omega$-hydroxyl metabolites 19- and 20-HETE. ARA is metabolized to four biologically active EET regioisomers, the 5,6-EET, 8,9-EET, 11,12-EET, and 14,15- EET. All EETs are then further metabolized into less active dihydroxy-eicosatrienoic acids (DHETs) by the enzyme soluble epoxide hydrolase (sEH; EC 3.3.2.10) (Morisseau and Hammock, 2013).

Existing drugs target the COX and LOX branches of the ARA cascade (Marnett, 2009; Haeggström et al., 2010). These branches largely synthesize proinflammatory mediators such as the prostanoids and leukotrienes. Most recently discovered branch, the cytochrome P450 branch, however, has not been adequately exploited as a pharmaceutical target. This branch produces both anti- and to lesser degree proinflammatory metabolites, including the 19- and 20-HETE (Williams et al., 2010). Notably, anti-inflammatory epoxy fatty acids such as EETs and epoxydocosapentaenoic acids (EDPs) are produced by cytochrome P450s (Spector, 2009; Imig, 2012). EETs seem to promote the resolution of inflammation, rather than prevent, in a manner similar to that exhibited by mediators in the LOX pathway (Serhan et al., 2011). EETs reduce inflammation, but are also analgesic, antifibrotic, and antihypertensive, acting in both paracrine and autocrine fashion (Spector, 2009; Bettaieb et al., 2015; Harris et al., 2015). However, the in vivo instability of EETs because of their rapid metabolism by $\mathrm{sEH}$ impeded understanding the roles of these lipid mediators until the development of potent and orally available $\mathrm{sEH}$ pharmacological inhibitors became available (Chacos et al., 1983; Morisseau et al., 1999).

The hydrolysis of EETs to DHETs by sEH was considered as an inactivation process in which bioactive metabolites were degraded to inactive products (Schmelzer et al., 2005). However, recent findings suggest that DHETs are also bioactive and are proinflammatory (Norwood et al., 2010), as are the diols linoleate epoxides (Viswanathan et al., 2003). Thus, sEH inhibition might be a powerful approach for reducing inflammation not only by stabilizing the anti-inflammatory mediators, but also by reducing proinflammatory mediator production. Furthermore, sEH inhibitors act in synergy with existing anti-inflammatory drugs, including COX and LOX inhibitors (Schmelzer et al., 2006; Liu et al., 2010), as well as anti-inflammatory phosphodiesterase inhibitors (Inceoglu et al., 2011).

The current study aimed to test the potential utility of $\mathrm{sEH}$ pharmacological inhibition in periodontitis, in which we evaluated the effects of sEH inhibition and EETs on bone loss using a mouse model of bacterial periodontitis.

\section{Materials and Methods}

Aggregatibacter actinomycetemcomitans (JP2) was purchased from American Type Culture Collection (Manassas, VA) and cultured inhouse under anaerobic conditions to ensure viability and virulance.

Chemicals. The sEH inhibitor 1-(1-propanoylpiperidin-4-yl)-3-[4(trifluoromethoxy)phenyl]urea was synthesized in-house, purified, and chemically characterized, as described earlier (Rose et al., 2010). The methyl ester of arachidonic acid was from NuChek Prep (Elysian, MN). Chromatography grade solvents for high-pressure liquid chromatography were obtained from Fisher Scientific (Pittsburgh, PA). Standards for Liquid chromatography tandem-mass spectrometry (LC-MS/MS) were purchased from Cayman Chemical (Ann Arbor, MI). The EET methyl esters were synthesized, purified, and characterized in-house using procedures published previously (Morisseau et al., 2010). The final regioisomeric mixture was analyzed using LC-MS/MS to ensure purity and regioisomeric ratio, which was 2.2:1.6:1.1:1, for 14, 15-:11, 12-:8, 9-:5, and 6-EpETrE, respectively.

Bacterial Viability. The potential bacteriostatic or bactericidal effects of trifluoromethoxyphenyl-3-(1-propionylpiperidine-4-yl) urea (TPPU) at the administered dose were tested in vitro. The microbial inoculum of $A$. actinomycetemcomitans was prepared and adjusted to $5 \times 10^{6}$ colony-forming units/mL in tryptic soy broth. TPPU was then dissolved to a final concentration of $10 \mu \mathrm{M}$ in polyethylene glycol (PEG)400 (Fisher Scientific, Nidderau, Germany) and was added and incubated at $37^{\circ} \mathrm{C}$ for 24 hours anaerobically. PEG400 solution without the inhibitor was used as a control. Experiments were performed in duplicates on 3 different days.

Animals and Animal Care. C57BL/6 (wild-type) and $\mathrm{sEH}^{-/-}$ [knockout (KO)] male mice were maintained under standard conditions, $23 \pm 1^{\circ} \mathrm{C}, 12$-hour light-dark cycle, ad libitum food, and water in housing facilities at University of California, Davis. Animals were agematched, and each group consisted of eight mice, 6-7 weeks old and weighing $20-25 \mathrm{~g}$. All procedures were in agreement with standards for the care of laboratory animals, as outlined in the National Institutes of Health Guide for the Care and Use of Laboratory Animals. All procedures were performed according to institutional guidelines for animal experimentation and were approved by the Animal Resource Services of the University of California, Davis, which is accredited by the American Association for the Accreditation of Laboratory Animal Care.

Periodontitis Model and Treatments. Animals orally received $1 \times 10^{9}$ colony-forming units/ml diluted culture of freshly grown $A$. actinomycetemcomitans JP2, in a volume of $100 \mu \mathrm{l}$ phosphate-buffered saline containing $2 \%$ carboxymethylcellulose. The solution was placed into the oral cavity with a micropipette, and the procedure was repeated at 48 and 96 hours after the first inoculation. Treatment with TPPU and EETs was initiated after the third inoculation of bacteria. The inhibitor was dissolved in PEG400 and administered at $1 \mathrm{mg} / \mathrm{kg} / \mathrm{d}$ by oral gavage. For the treatment with EETs, we attempted to apply viscous solution of mixture of EET methyl ester regioisomers dissolved in PEG400 to the gum tissue using a fine pipette at a dose of $1 \mu \mathrm{g} / \mathrm{kg} / \mathrm{d}$. The mice received the entire EET dose. This was done immediately before the oral gavage with both groups of mice receiving TPPU or mice receiving vehicle alone. Because EETs are unstable in stomach acid, we assume exposure is largely topical or buccal. All treatments continued for 15 days. The plasma and blood concentration of TPPU was quantified, as previously described, by LC-MS/MS analysis (Ostermann et al., 2015). The negative control group consisted of uninfected mice (sham-infected); the positive control group was infected and received the vehicle only. One day after the treatment period (16th day), animals were sacrificed by cardiac puncture after anesthesia with xylazine/ketamine. Plasma and whole blood were sampled and frozen until analysis. 
Quantification of Alveolar Bone Loss. Evaluation of alveolar bone loss was performed, as described previously (Eskan et al., 2012; Napimoga et al., 2013). Sixteen days after the third inoculation, animals were sacrificed, and the jaws were removed and defleshed, then immersed overnight in $3 \%$ hydrogen peroxide, and stained with $1 \%$ methylene blue in phosphate-buffered saline. Horizontal bone loss was assessed morphometrically by measuring the distance between the cement-enamel junction and the alveolar bone crest of the first and second molars. Measurements at 14 buccal sites per mouse ( 7 sites each on the left and right maxillary molars) were made under a microscope, pictures were taken, and bone measurements were analyzed using the Image $\mathrm{J}$ software suit. Random and blinded measurements were taken by the same calibrated person (C.A.T.-d.-S.). Intraexaminer reproducibility of the measurements achieved $>90 \%$. Because we elected to use toluidine blue staining, other approaches of histology were not pursued mainly because of a limited availability of experimental animals.

Western Blotting. Western blotting was performed, as described earlier (Bettaieb et al., 2013). Briefly, tissues were lysed first and clarified by centrifugation, and protein concentrations were determined using the bicinchoninic acid protein assay kit (ThermoFisher Scientific, Waltham, MA). Equal amounts of protein $(20 \mu \mathrm{g})$ from the gingival tissue were resolved by SDS-PAGE and transferred to polyvinylidene difluoride membranes. Immunoblotting of lysates was performed with antibodies for monocyte chemotactic protein 1 (MCP-1; BioLegend, San Diego, CA), epidermal growth factor-like module-containing mucin-like hormone receptor-like 1 (F4/80), binding immunoglobulin protein, phosphorylated p38-pp38 ( $\left.\mathrm{Thr}^{180} / \mathrm{Tyr}^{182}\right)$, p38 mitogen-activated protein kinases (p38), phosphorylated c-Jun N-terminal kinase (JNK; $\mathrm{Thr}^{183} / \mathrm{Tyr}^{185}$ ), JNK or cleaved caspase-3 (Cell Signaling Technology, Beverly, MA), receptor activator of nuclear factor-кB (RANK), RANK ligand (RANKL), osteoprotegerin (OPG), or $\alpha$-tubulin (Santa Cruz Biotechnology, Santa Cruz, CA). Antibodies for sEH were generated in-house using recombinantly expressed mouse sEH following affinity purification. After incubation with the appropriate secondary antibodies, proteins were visualized using Luminata Fort Western HRP substrate (Millipore, Billerica, MA). Pixel intensities of immunoreactive bands were quantitated using FluorChem Q Imaging software (Alpha Innotech, CA). For phosphorylated proteins, data were presented as phosphorylation level normalized to total protein expression, and, for nonphosphorylated proteins, as total protein expression normalized to $\alpha$-tubulin.

Statistical Analysis. The statistical analyses were performed using Prism 5.0 (GraphPad, San Diego, CA) or the SigmaPlot Software Suite (Systat, San Jose, CA). The data were first examined for normality using the Kolmogorov-Smirnov test, and then analyzed using one-way analysis of variance (ANOVA). If there was a significant difference among-subjects main effect for the treatment group following one-way ANOVA, or one-way ANOVA on ranks, post hoc contrasts, using the Student's Newman-Keuls multiple comparison or Tukey's all pairwise post hoc tests, as automatically suggested by the software suit. Data are presented as mean \pm S.E.M.

\section{Results}

TPPU Does Not Affect Bacterial Viability. In the present study, we tested the potential effects of TPPU, a potent and selective pharmacological inhibitor of sEH, to inhibit bone loss caused by periodontal disease (PD) in mouseinduced periodontitis model. TPPU or its vehicle did not have bacteriostatic or bactericidal effect on the cultures used to induce the PD. As demonstrated in Supplemental Fig. 1, no change in the growth of the A. actinomycetemcomitans was observed in the presence or absence of TPPU.

Chemical Inhibition of sEH Reduces Bone Loss. There were no A. actinomycetemcomitans in the oral cavities of mice prior to deliberate infection. In contrast, persistent oral colonization by the pathogen was confirmed in all infected animals on the last day. Next, bone loss was quantified in all mice (Fig. 1A). Sham-infected animals presented the lowest distance between cement-enamel junction and alveolar bone crest during the experimental period (Fig. 1B). In contrast, animals orally infected with $A$. actinomycetemcomitans but receiving no treatment showed significantly greater bone loss when compared with the uninfected animals (Fig. 1C). Animals infected and orally treated with EET methyl esters at a dose of $1 \mu \mathrm{g} / \mathrm{kg} / \mathrm{d}$ did not display a significant reduction in bone loss (Fig. 1D). In contrast, mice treated with TPPU had significantly lower bone loss than the untreated infected animals and were comparable to the levels of uninfected animals (Fig. 1E). Moreover, consistent with this observation, the mice that received combination of TPPU and EET methyl esters had significantly lower bone loss compared with untreated infected animals (Fig. 1F). However, this decrease was not significantly more than the TPPU group $(P=0.53)$. Using this dosing scheme, one would expect a near-complete and sustained inhibition of $\mathrm{sEH}$ activity. Blood concentration of TPPU at the end of the experiment also supports the argument that the target enzyme is significantly inhibited. Groups of mice that received TPPU and TPPU + EET methyl esters had more than $8 \mu \mathrm{g} / \mathrm{mL}$ TPPU detected in the blood, even after 24 hours of the last oral administration (Supplemental Fig. 2). There were no differences regarding the blood TPPU concentration in the group that received TPPU + EET methyl esters. These levels reiterate the argument that the target enzyme is significantly inhibited (Liu et al., 2009, 2010; Rose et al., 2010). Thus, in these studies, TPPU was sufficiently stable in vivo

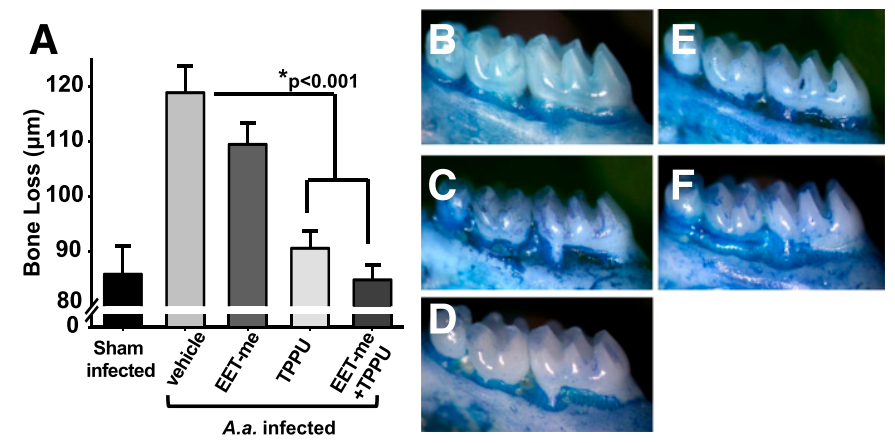

Fig. 1. Inhibition of sEH with a potent and orally available smallmolecule TPPU decreases bone loss. Mice were inoculated with freshly grown cultures of A. actinomycetemcomitans on 3 consecutive days, as described in Materials and Methods. The vehicle PEG400, TPPU ( $1 \mathrm{mg} / \mathrm{kg})$, and EET methyl esters ( $1 \mu \mathrm{g} / \mathrm{kg}$ diluted in PEG400) were orally administered daily by gavage. Treatments continued until 15th day postinfection, and samples were obtained on the 16th day. Distance $(\mu \mathrm{m})$ between the cement-enamel junction and the alveolar bone crest for all experimental groups was quantified. (A) The group vehicle alone developed significant bone loss, whereas groups treated with TPPU and TPPU + EET methyl esters displayed a marked reduction in bone loss. Mice receiving EET methyl esters were not different than vehicletreated infected mice, suggesting the necessity of inhibiting $\mathrm{sEH}$ for EETs to display activity. (B-F) Panels display deboned and methylene blue-stained teeth from sham $(\mathrm{B}, n=8)$, mice infected with A. actinomycetemcomitans (C, $n=14)$, EET methyl ester ( $1 \mu \mathrm{g} / \mathrm{kg} / \mathrm{daily})$ treated (D, $n=12$ ), TPPU, $1 \mathrm{mg} / \mathrm{kg}$ treated (E, $n=13$ ), and EET methyl ester + TPPU-treated groups $(\mathrm{F}, n=13)$. The dark stained areas indicate sites of bone loss. The results are expressed as mean \pm S.E.M. $(* P<0.001$, one-way ANOVA followed by Student's Newman-Keuls post hoc all pairwise comparison.) 
to provide effective concentrations throughout the course of the experiment.

Genetic Ablation of sEH Recapitulates the Effects of sEH Inhibitor on Bone Loss. To support the results of sEH pharmacological inhibition, we performed similar experiment using sEH global $\mathrm{KO}$ mice. Consistent with the results obtained earlier, wild-type mice infected with $A$. actinomycetemcomitans showed significantly greater bone loss when compared with the uninfected animals (Fig. 2). Remarkably, infected sEH KO mice displayed highly significant reduction in bone loss, similar to the levels of uninfected group (Fig. 2, D and E). Findings using $\mathrm{sEH} \mathrm{KO}$ mice recapitulate observations from sEH pharmacological inhibition using TPPU.

Inhibition of sEH Alters Key Regulators of Bone Remodeling. To understand the basis of these effects, we determined the levels of key regulators of bone remodeling, a highly dynamic process. The RANK/RANKL/OPG system is generally accepted as a master regulator of bone loss and regeneration (Lacey et al., 1998). RANKL synthesized by osteoblasts, cells that synthesize new bone, targets RANK on the osteoclasts, cells that resorb bone. This stimulates bone loss by activating osteoclasts. The third arm of the system is osteoblast-derived OPG, which is a soluble decoy receptor for RANKL and prevents its binding to RANK and thereby finetuning bone remodeling. As expected, in infected control mice, levels of RANK, RANKL, OPG, and MCP-1 in the gum tissue were increased, compared with uninfected animals (Fig. 3). This reiterates the imbalance in bone-remodeling process as well as increased infiltration of cells that mediate inflammation, arguments supported by data presented in this work. Similarly, the levels of infiltrating macrophages that are $\mathrm{F} 4 / 80^{+}$displayed a drastic increase in the $\mathrm{PD}$ group. In contrast, TPPU treatment significantly reduced the expression of these biomarkers. Consistent with the TPPU group, animals treated with TPPU + EET methyl esters $(1 \mu \mathrm{g} / \mathrm{kg})$ displayed a nearly identical profile in the expression of all five proteins quantified (Fig. 3, B-F). Equally importantly, in sEH $\mathrm{KO}$ mice infected with $A$. actinomycetemcomitans, the effects

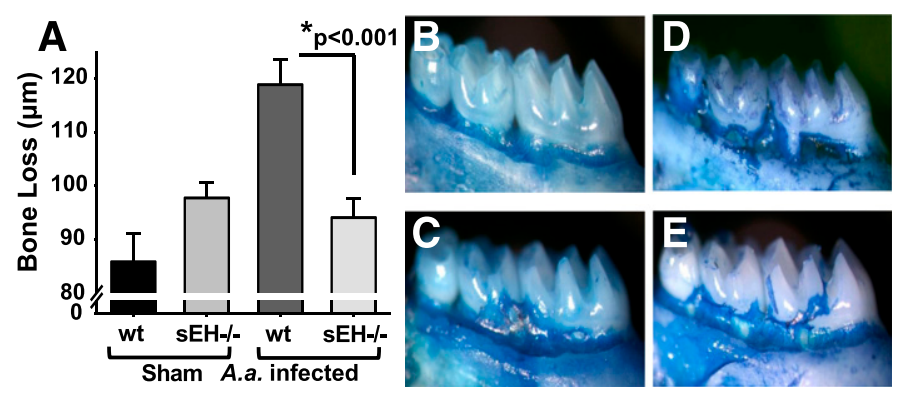

Fig. 2. Genetic inhibition of sEH by gene KO decreases bone loss similar to chemical inhibitor. $\mathrm{sEH}^{-/-}$and wild-type $\mathrm{C} 57 \mathrm{BL} / 6$ mice were from a University of California, Davis-maintained colony. Mice at age 6 weeks were infected with $A$. actinomycetemcomitans three consecutive times, as described for Fig. 1, and at the end of the treatment period distance $(\mu \mathrm{m})$ between the cement-enamel junction and the alveolar bone crest for all experimental groups was quantified. In parallel to the results with the sEH inhibitor TPPU, the genetic KO of $\mathrm{sEH}$ resulted in significantly reduced bone loss (Panel A). Panels display wild-type sham-infected (B, $n=8), \mathrm{sEH}^{-/-}$sham-infected (C, $\left.n=15\right)$, wild-type mice orally infected with $A$. actinomycetemcomitans ( $\mathrm{D}, n=14$ ), and $\mathrm{sEH}^{-1-}$ mice orally infected with $A$. actinomycetemcomitans ( $\mathrm{E}, n=14)$. The dark stained areas indicate sites of bone loss. The results are expressed as mean \pm S.E.M. ( $* P<0.001$, one-way ANOVA followed by Student's Newman-Keuls post hoc all pairwise comparison). wt, wild type. of TPPU treatment were recapitulated. More specifically, in sEH KO mice, levels of RANK, RANKL, MCP-1, and F4/80 were similar to TPPU-treated group, whereas they displayed slightly higher levels of OPG (Fig. 3, B-E). However, the higher levels of OPG could be seen as an advantage because this would lead to less bone loss. Overall, these results underline the accelerated and incongruent bone remodeling in $\mathrm{PD}$ and that these pathologic changes can be recalibrated by sEH deletion or pharmacological inhibition.

The significant reduction in MCP-1 expression is suggestive of a decrease in inflammatory cell migration and therefore inflammation. Therefore, we further monitored key downstream kinases known to be phosphorylated in response to inflammatory signals. Two stress kinases, p38 and JNK, activate their respective signaling cascades and increase inflammation, cytokine synthesis, and apoptosis. In the gingival tissue of infected mice, levels of phosphorylated JNK and pp38 (phosphorylated p38) were largely increased, supporting the idea that dysregulated host responses have a pivotal role in PD. In contrast, in treatment with TPPU, TPPU + EET methyl esters, or in sEH KO mice infected with A. actinomycetemcomitans, phosphorylation of both kinases was greatly reduced (Fig. 4). Concomitant to these changes, the drastic decrease in the levels of macrophages quantified by F4/80 levels by the treatments supports the inflammationreducing effects of inhibition of $\mathrm{sEH}$. These observations are consistent with the argument that $\mathrm{sEH}$ inhibition dampens inflammatory response in PD.

The sEH is a significant regulator of endoplasmic reticulum (ER) stress response (Bettaieb et al., 2013, 2015; Harris et al., 2015). Therefore, we evaluated whether ER stress-mediated pathways are active in PD and if SEH deletion or pharmacological inhibition attenuates ER stress response. We observed the activation of the two major branches of the ER stress signaling cascade in the gingival tissue samples. Although the levels of phosphorylated protein kinase RNA-like ER kinase and phosphorylated inositol-requiring enzyme $1 \alpha$ were below the detection limit in untreated wild-type and sEH KO mice, they were significantly elevated in the gum tissue of mice infected with A. actinomycetemcomitans (Fig. 5). Consistent with their phosphorylation, downstream targets for each ER stress sensor (eukaryotic initiation factor $2 \alpha$ and spliced X-box binding protein 1) were significantly elevated in infected mice. Phosphorylated eukaryotic initiation factor $2 \alpha$ and spliced $\mathrm{X}$-box binding protein 1 were below the level of detection in healthy tissues but were significantly increased in mice with PD. Notably, sEH KO mice infected with A. actinomycetemcomitans displayed significantly lower ER stress compared with wild-type animals infected with $A$. actinomycetemcomitans. However, markers of ER stress were higher in $\mathrm{KO}$ mice compared with TPPU-treated wild-type animals. This observation suggests that chemical inhibition of $\mathrm{sEH}$ was more efficacious in attenuating ER stress. In contrast, there was no difference between TPPU alone or in the presence of EET methyl esters at the concentrations tested $(P>0.05)$.

A consequence of activation of ER stress sensors is a decrease in general protein synthesis and an increase in components that assist in overcoming stress. However, if these compensatory mechanisms fail to restore/maintain homeostasis, then the cells will engage apoptosis. To determine whether PD leads to intense inflammatory conditions and ER stress that compel the cells to activate apoptotic 

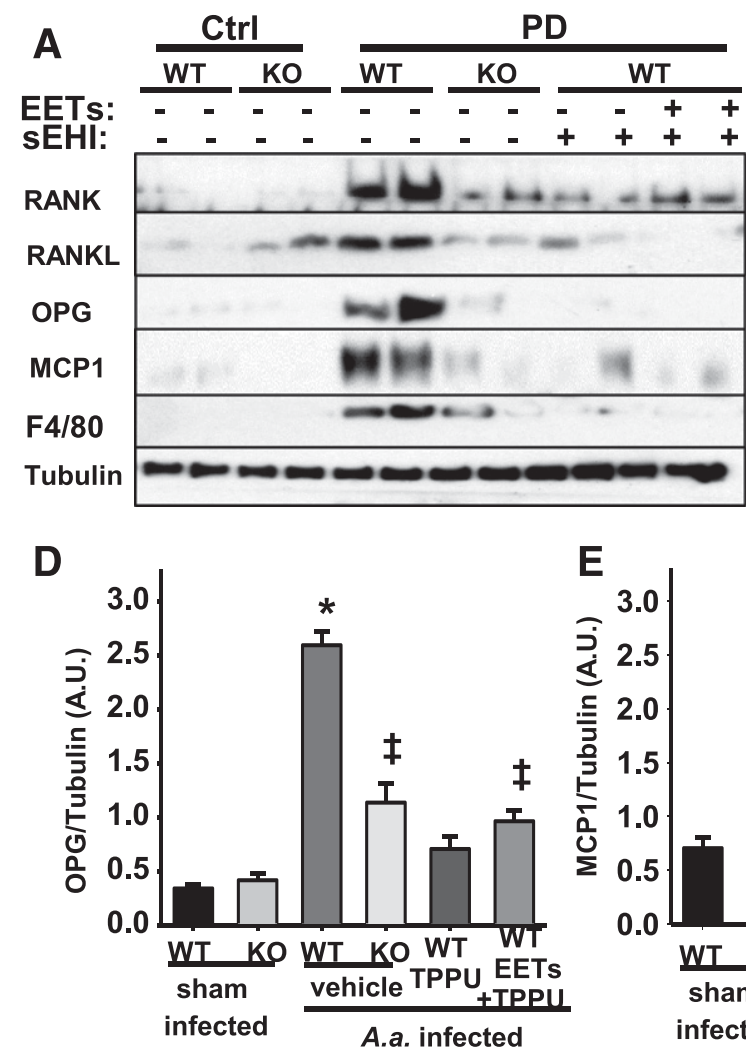

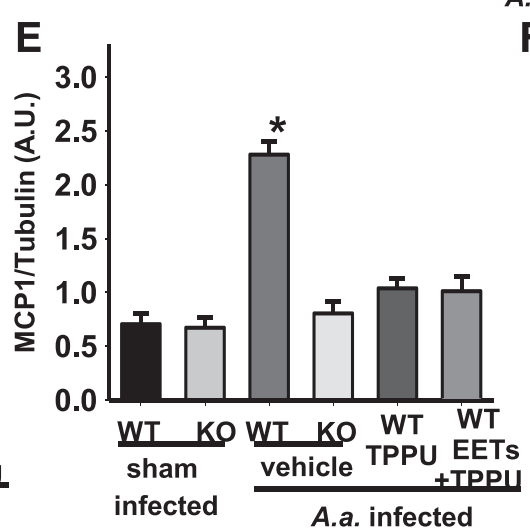

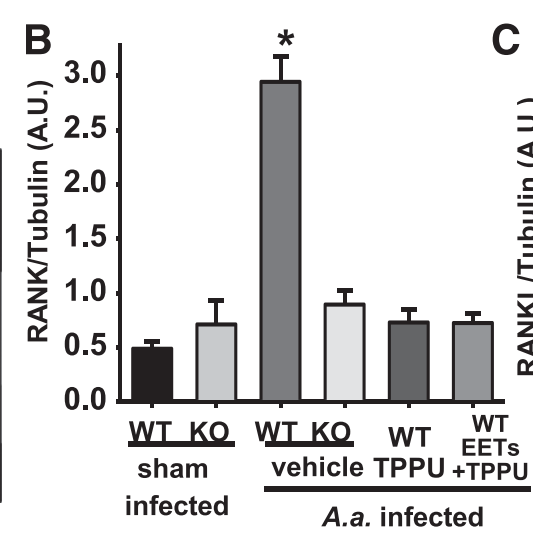

$\mathbf{F}$
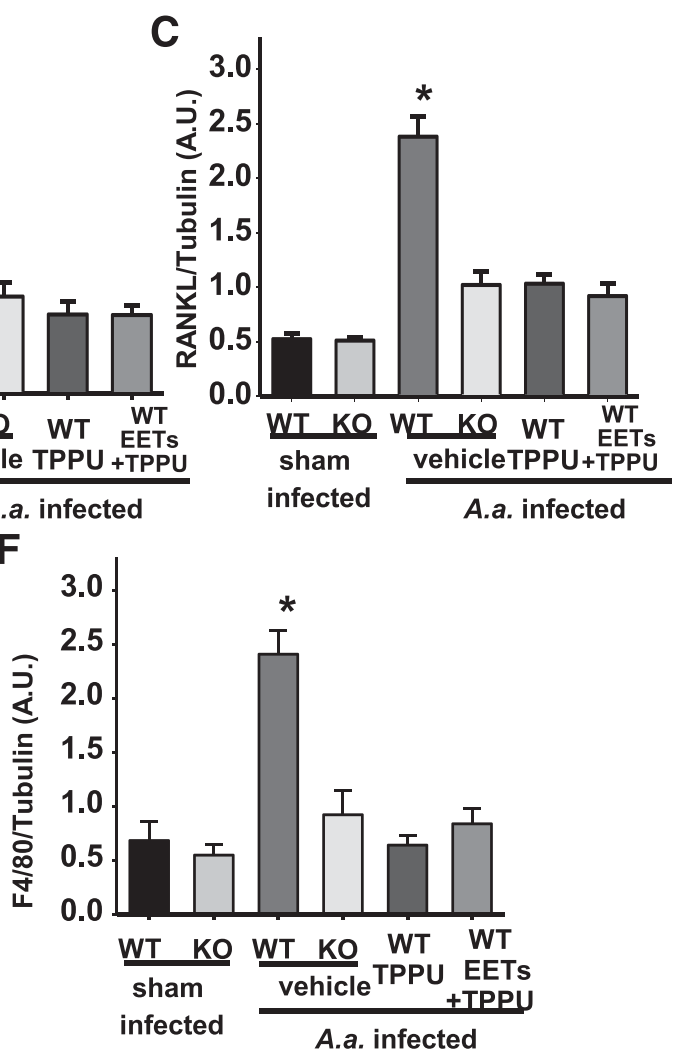

Fig. 3. The dysregulated RANK/RANKL/OPG system in PD is restored by chemical or genetic ablation of sEH. Protein expression levels of osteoclastogenesis-related factors in gingival tissues from all experimental groups were investigated by Western blotting. For quantification, band intensity was normalized to that of $\alpha$-tubulin. Protein band intensity is represented as arbitrary units. Density quantification included all animals, and mean \pm S.E.M. of each group ( $n=6$ per group) is displayed in the bar graphs. (A) Original blots displaying two randomly selected animals. (B) Bar graphs of mean band intensity for RANK (B), RANKL (C), OPG (D), MCP-1 (E) measured for all six mice and F4/80 (F) $(* P<0.001$, $¥ P<0.03$, one-way ANOVA followed by Student's Newman-Keuls post hoc all pairwise comparison). WT, wild type.

cascades, we monitored the levels of cleaved caspase-3 (Fig. 6). Caspase-3 is activated by upstream caspases and is an integrator and marker of activation of the apoptotic signaling pathway. c-Caspase-3 expression was exceedingly low under normal conditions, much like the other markers used in this study. However, infection by A. actinomycetemcomitans significantly increased the levels of c-Caspase-3 in all mice, but most remarkably in the vehicle control group (Fig. 6). This supports the hypothesis that activated ER stress in PD is linked to cell death. In contrast, mice treated with TPPU, TPPU + EET methyl esters, or the sEH KO mice displayed significantly lower levels of c-Caspase-3. This observation
A

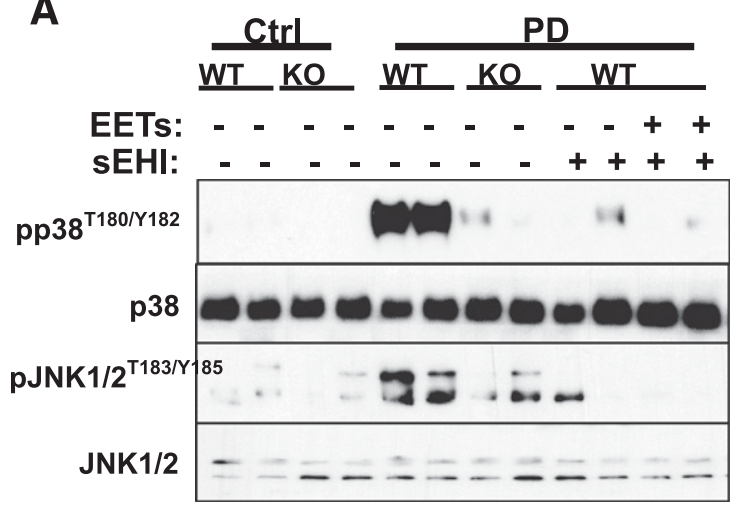

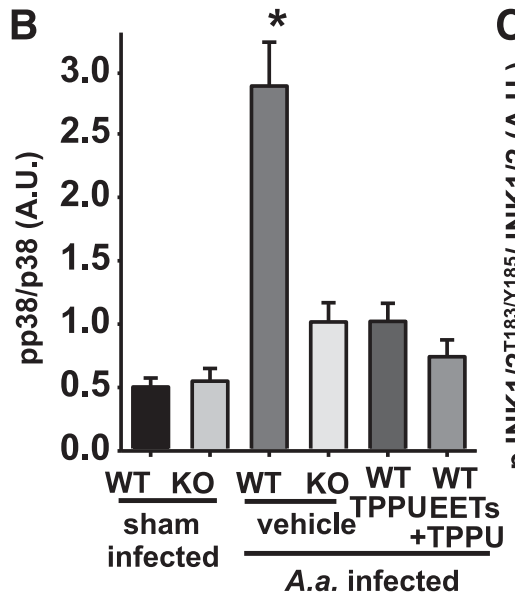

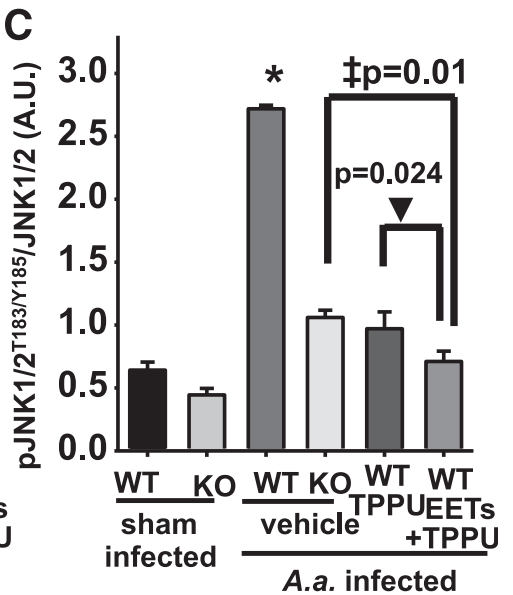

Fig. 4. PD-mediated phosphorylation of proinflammatory p38 and JNK1/2 is reduced by chemical or genetic ablation of sEH. Phosphorylation and activation of p38 and JNK1/2 were quantified from all groups by normalizing band intensity to that of $\alpha$-tubulin. (A) Original blots displaying two randomly selected animals. (B and C). Bar graphs of phosphorylation status of p38 and JNK1/2. Mean band intensity is measured for all six mice and is represented as arbitrary units (mean \pm S.E.M.) $(* P<0.001, \ddagger P=0.01, \nabla P=0.024$, one-way ANOVA followed by Student's Newman-Keuls post hoc all pairwise comparison). WT, wild type. 


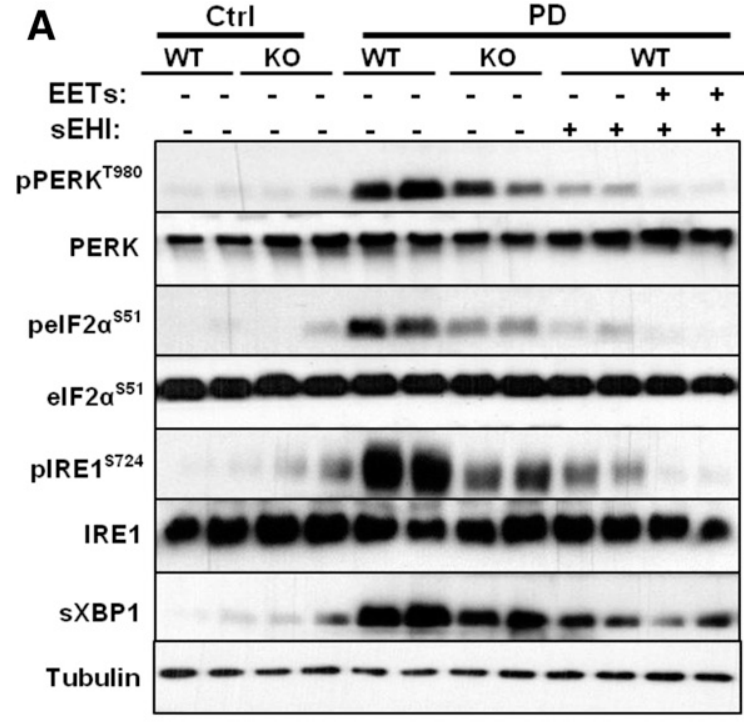

Fig. 5. ER stress sensors are activated in gingival tissues of mice with PD
selected animals for each group. (B-E) Bar graphs of phosphorylation stat
inositol-requiring enzyme 1 , and expression level of spliced X-box binding
measured for all six mice and is represented as arbitrary units (mean $\pm \mathrm{S}$.
ranks, followed by Tukey's all pairwise multiple comparison post hoc test).
suggests that inhibition of sEH was largely able to reduce ER
stress and the ensuing apoptosis. However, given the remaining 10-fold increase in c-Caspase-3 in treated groups, other inflammation-associated apoptotic signaling cascades may not have been targeted by inhibition of $\mathrm{sEH}$.

\section{Discussion}

The pathogenesis of PD is recognized as infection-induced inflammatory tissue destruction. At the site of tissue destruction, cytokines and inflammatory mediators are elevated. The prevalence of periodontitis in the United States adult population is estimated at over $47 \%$. In adults aged 65 and older, $64 \%$ had either moderate or severe stages of PD (Eke et al., 2012). This prevalence rate highlights the uniqueness of PD among other conditions. Therefore, efforts to understand the pathophysiology of the disease as well as different approaches to control it should result in tremendous health benefits. In the present study, we demonstrated that an inhibitor of sEH largely abrogates bone loss caused by PD. This seems to be based on the effects of inhibition of sEH to decrease key osteoclastogenic molecules, as well as lowering inflammation-triggered ER stress and associated apoptosis in the gingival tissue.

Epoxy fatty acid generation from ARA and other unsaturated fatty acids is the third and latest major branch of the ARA cascade. The epoxyeicosatrienoic acids seem to have mostly anti-inflammatory functions that are in contrast to proinflammatory products of the other two branches (Capdevila et al., 1981). Multiple cytochrome P450s form epoxy fatty acids, and ARA, for example, is converted into
5,6-EET, 8,9-EET, 11,12-EET, and 14,15-EET, all of which have biologic activity.

Early evidence on the anti-inflammatory effects of 11,12EET by preventing tumor necrosis factor- $\alpha$-induced activation of nuclear factor $\kappa \mathrm{B}$ and the subsequent increase in
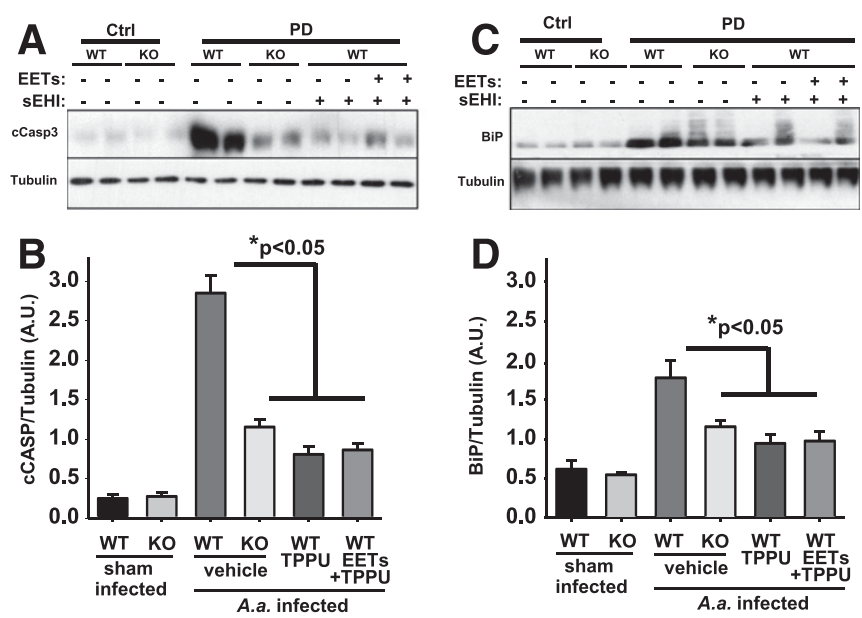

Fig. 6. Genetic ablation or chemical inhibition of $\mathrm{sEH}$ reduces apoptosis in gingival tissue of mice infected with A. actinomycetemcomitans. Original blot displaying two randomly selected animals for each group. (A) Expression level of c-Caspase-3 and (C) immunoglobulin binding protein $(\mathrm{BiP})$ is quantified by measuring band intensity for all six mice for each group and normalized to the expression level of a-tubulin. (B and D) Bar graph of mean caspase-3 and BiP band intensity respectively, represented as arbitrary units (mean 6 S.E.M.) $(* P<0.05$, Kruskal-Wallis one-way analysis of variance on ranks, followed by Tukey's all pairwise multiple comparison post hoc test). sEHI, soluble epoxide hydrolase inhibitor; WT, wild type. 
vascular cell adhesion molecule-1 expression in mice is recently supported by other studies (Node et al., 1999; Chiamvimonvat et al., 2007). However, in vivo, all EET regioisomers are degraded quickly by $\mathrm{sEH}$, leading to the production of metabolites known as DHETs. Thus, in the absence of a sEH inhibitor, most of the biologic effects of EETs are difficult to observe. The potent inhibitor of sEH TPPU stabilizes the EETs and other epoxy fatty acids in vivo and promotes anti-inflammatory processes. The presumed mechanism of the therapeutic effect seems to involve homeostatic regulation of the RANK, RANKL, and OPG system, a master regulator of osteoclastogenesis. Furthermore, inhibition of sEH also decreases the chemokine MCP-1, as would be expected from the anti-inflammatory effects of $\mathrm{sEH}$ inhibition. Overall, results are nearly identical across chemical inhibitor and global KO mice, highly supportive of the idea that inhibition of sEH rather than inhibitor structures specific pharmacological efficacy.

Another line of evidence reported recently also argues for the activity of EETs to prevent bone loss in a different experimental paradigm (Guan et al., 2015). In an ovariectomyinduced mouse model of bone loss, administration of EETs seems to prevent bone loss through a similar mechanism in which EETs normalize the plasma RANKL to OPG ratio, whereas RANK levels were not reported. Instead, authors demonstrate suppression of RANK by the free acid forms of EETs, in bone marrow mononuclear cells and the RAW264.7 cell line when induced by treating the cells with RANKL. In the ovariectomized mice, the prominent mechanism of bone loss seems to be driven by decrease in OPG. In contrast, we report the levels of RANK, RANKL, and OPG are all increased in gingival tissue by approximately sixfold in response to inflammation. These differences highlight the mechanistic distinction between the ovariectomy- and PD-induced bone loss models. Remarkably, in TPPUtreated and sEH KO mice in our study, RANK, RANKL, and OPG were normalized to nearly preinfection levels, suggesting a potential therapeutic effect. This is, of course, an expected difference from the earlier study given that EETs have short in vivo half-lives and inhibition of $\mathrm{sEH}$ stabilizes these molecules by preventing their degradation by the sEH enzymatic activity. TPPU is easy to administer orally or in drinking water, yields high exposure, and is a potent inhibitor of sEH (Rose et al., 2010). It is also important to highlight that, in our study, administration of EET methyl esters alone topically and directly onto the periodontal tissue did not prevent the inflammatory periodontal bone loss. However, the EET methyl esters in the presence of TPPU displayed detectable changes in markers of ER stress (Fig. 5). Overall, the findings demonstrate that inhibition of $\mathrm{sEH}$ is a viable approach to treat complex conditions that include progressive bone loss.

An active periodontal lesion is characterized by the prominent infiltration of B and T cells (Okada et al., 1983). Adoptive transfer of $\mathrm{RANKL}^{+}$, antigen-specific T cells induces bone loss in rat periodontal tissue that received local injection of the T cell antigen. Furthermore, $\mathrm{T}$ and $\mathrm{B}$ cells are likely the major sources of RANKL in the inflamed gingival tissues (Kawai et al., 2006). A benefit of inhibiting the sEH in this study was the sharp decrease in tissue MCP-1 (CC chemokine ligand-2) level. The potent chemoattractant MCP-1 is largely seen as the driver of monocyte infiltration into the gingival tissue. In support of this possibility, levels of $\mathrm{F} 4 / 80^{+}$macrophages increased by about fivefold in the $\mathrm{PD}$ group and were completely returned to healthy levels in all the treatment groups, including in the $\mathrm{sEH}^{-/-}$mice. Thus, it is plausible that administration of TPPU or in the sEH KO mice lower levels of MCP-1 decreased the infiltration of inflammatory cells and thereby led to the downregulation of the RANK/RANKL/OPG axis (Fig. 7). However, it seems more likely that EETs also had a direct effect on the RANK/RANKL/OPG axis because, whereas markers of ER stress were mostly normalized, expression of RANK, RANKL, and OPG was fully restored to preinfection levels. This observation suggests that decreased inflammatory cell infiltration is a distinct effect of $\mathrm{sEH}$ inhibition and is an additional benefit. The multiple mechanisms responsible for $\mathrm{sEH}$ inhibition-mediated decrease in PD are summarized in Fig. 7. As much as inflammation is drastically blocked by inhibition of $\mathrm{sEH}$, the decrease in ER stress in these tissues may enable the cells to divert their resources to normalize homeostasis and thus not go toward activation of apoptotic responses.

Deletion of sEH and its pharmacological inhibition led to attenuation of the ER stress response in several experimental models (Bettaieb et al., 2013; Harris et al., 2015; Inceoglu et al., 2015). This homeostatic mechanism is involved in numerous pathologic conditions, including viral and bacterial infection and neurodegenerative diseases (Cao et al., 2016). The ER stress system is typically activated in response to stressors, including excessive amounts of unfolded proteins in the ER. ER stress response in PD was recently reported (Domon et al., 2009; Kung et al., 2015; Yamada et al., 2015). These authors suggested that modulation of the ER stress system could have therapeutic effects. Consistent with their prediction, in this study we detected the activation of ER stress in the gingival tissue, suggesting that, at the least, ER stress contributed to the development and progression of PD. The markers of activated ER stress were attenuated by $\mathrm{sEH}$ pharmacological inhibition and deletion. Activated ER stress signaling may lead to apoptosis when cells are unable to maintain homeostasis. This was reported in the gingival tissue of diabetic rats with PD (Kang et al., 2012; Cueno et al., 2016; Seo et al., 2016). In this study, we demonstrate the activation of apoptosis in conjunction with ER stress.

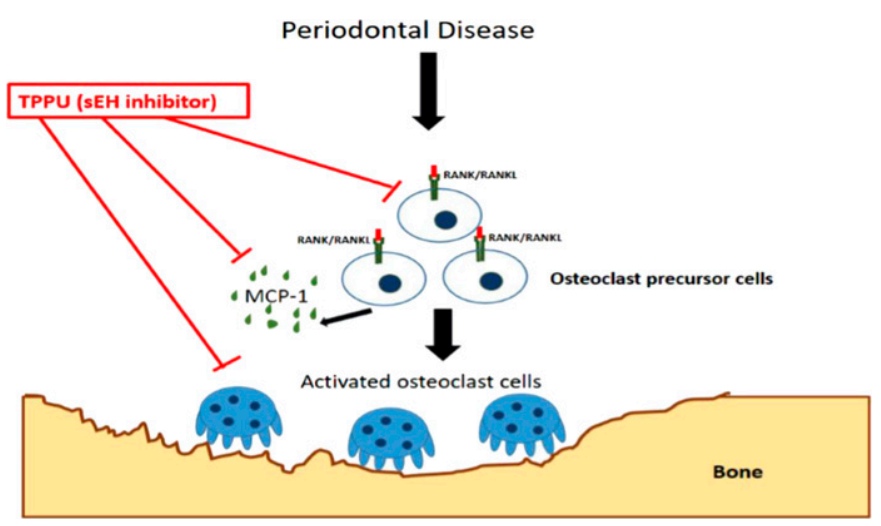

Fig. 7. Simplified schematic representation of $\mathrm{sEH}$ inhibition mediated suppression of inflammation and bone loss. Soluble epoxide hydrolase pharmacological inhibition decreases alveolar bone loss by modulating host inflammatory response, RANK-related signaling, ER stress, and apoptosis. 
However, blocking ER stress and inflammation with TPPU was sufficient to prevent apoptosis, and this was consistently observed in $\mathrm{sEH}^{-/-}$mice inoculated with $A$. actinomycetemcomitans. These findings are consistent with those reported earlier for PD models. Specifically, in diabetic rats, A. actinomycetemcomitans induced a caspase-3-dependent response and led to increased number of cells going through apoptosis in the gingival epithelial and connective tissues and increased bone loss (Kang et al., 2012).

Overall, the results reiterate the importance of the RANK/ RANKL/OPG system and its crosstalk with ER stress signaling in PD. These interactions ultimately give rise to increased apoptosis in the gingival tissue and bone loss. Given the importance and the epidemiology of gingival diseases, therapeutics that selectively targets the elements of the host inflammatory responses should prove useful to improve oral health. Our findings strongly indicate that inhibition of $\mathrm{sEH}$ is one such therapeutic approach. Nearly identical results from a small-molecule inhibitor of sEH and the mice with genetic $\mathrm{KO}$ of $\mathrm{sEH}$ support the idea that positively altering the bioactive lipid mediators, including EETs, is a viable approach to dampen destructive inflammation, apoptosis, and bone loss in PD.

\section{Authorship Contributions}

Participated in research design: Trindade-da-Silva, Bettaieb, Napimoga, Lee, Inceoglu, Ueira-Vieira, Haj, Hammock.

Conducted experiments: Trindade-da-Silva, Bettaieb, Lee, Bruun, Goswami.

Contributed new reagents or analytic tools: Lee, Bruun.

Performed data analysis: Trindade-da-Silva, Bettaieb, Napimoga,

Lee, Inceoglu, Ueira-Vieira, Bruun, Goswami, Haj, Hammock.

Wrote or contributed to the writing of the manuscript: Trindadeda-Silva, Bettaieb, Napimoga, Lee, Inceoglu, Ueira-Vieira, Bruun, Goswami, Haj, Hammock.

\section{References}

Bartold PM and Van Dyke TE (2013) Periodontitis: a host-mediated disruption of microbial homeostasis: unlearning learned concepts. Periodontol 2000 62: 203-217.

Bettaieb A, Chahed S, Bachaalany S, Griffey S, Hammock BD, and Haj FG (2015) Soluble epoxide hydrolase pharmacological inhibition ameliorates experimental acute pancreatitis in mice. Mol Pharmacol 88:281-290.

Bettaieb A, Nagata N, AbouBechara D, Chahed S, Morisseau C, Hammock BD, and Haj FG (2013) Soluble epoxide hydrolase deficiency or inhibition attenuates diet-induced endoplasmic reticulum stress in liver and adipose tissue. J Biol Chem 288:14189-14199.

Cao SS, Luo KL, and Shi L (2016) Endoplasmic reticulum stress interacts with inflammation in human diseases. J Cell Physiol 231:288-294.

Capdevila J, Chacos N, Werringloer J, Prough RA, and Estabrook RW (1981) Liver microsomal cytochrome P-450 and the oxidative metabolism of arachidonic acid. Proc Natl Acad Sci USA 78:5362-5366.

Chacos N, Capdevila J, Falck JR, Manna S, Martin-Wixtrom C, Gill SS, Hammock BD, and Estabrook RW (1983) The reaction of arachidonic acid epoxides (epoxyeicosatrienoic acids) with a cytosolic epoxide hydrolase. Arch Biochem Biophys 223:639-648.

Chiamvimonvat N, Ho CM, Tsai HJ, and Hammock BD (2007) The soluble epoxide hydrolase as a pharmaceutical target for hypertension. J Cardiovasc Pharmacol 50:225-237.

Cueno ME, Saito Y, and Ochiai K (2016) Periodontal disease level-butyric acid amounts locally administered in the rat gingival mucosa induce ER stress in the systemic blood. Microb Pathog 94:70-75.

Domon H, Takahashi N, Honda T, Nakajima T, Tabeta K, Abiko Y, and Yamazaki K (2009) Up-regulation of the endoplasmic reticulum stress-response in periodontal disease. Clin Chim Acta 401:134-140.

Eke PI, Dye BA, Wei L, Thornton-Evans GO, and Genco RJ; CDC Periodontal Disease Surveillance workgroup: James Beck (University of North Carolina, Chapel Hill, USA), Gordon Douglass (Past President, American Academy of Periodontology), Roy Page (University of Washington) (2012) Prevalence of periodontitis in adults in the United States: 2009 and 2010. J Dent Res 91: 914-920.

Eskan MA, Jotwani R, Abe T, Chmelar J, Lim JH, Liang S, Ciero PA, Krauss JL, Li F, Rauner M, et al. (2012) The leukocyte integrin antagonist Del-1 inhibits IL-17mediated inflammatory bone loss. Nat Immunol 13:465-473.

Flemmig TF (1999) Periodontitis. Ann Periodontol 4:32-38.
Guan H, Zhao L, Cao H, Chen A, and Xiao J (2015) Epoxyeicosanoids suppress osteoclastogenesis and prevent ovariectomy-induced bone loss. FASEB $J$ 29: 1092-1101.

Haeggström JZ, Rinaldo-Matthis A, Wheelock CE, and Wetterholm A (2010) Advances in eicosanoid research, novel therapeutic implications. Biochem Biophys Res Commun 396:135-139.

Hajishengallis G, Darveau RP, and Curtis MA (2012) The keystone-pathogen hypothesis. Nat Rev Microbiol 10:717-725.

Harris TR, Bettaieb A, Kodani S, Dong H, Myers R, Chiamvimonvat N, Haj FG, and Hammock BD (2015) Inhibition of soluble epoxide hydrolase attenuates hepatic fibrosis and endoplasmic reticulum stress induced by carbon tetrachloride in mice. Toxicol Appl Pharmacol 286:102-111.

Hasturk H, Kantarci A, Goguet-Surmenian E, Blackwood A, Andry C, Serhan $\mathrm{CN}$, and Van Dyke TE (2007) Resolvin E1 regulates inflammation at the cellular and tissue level and restores tissue homeostasis in vivo. J Immunol 179: 7021-7029.

Imig JD (2012) Epoxides and soluble epoxide hydrolase in cardiovascular physiology. Physiol Rev 92:101-130.

Inceoglu B, Bettaieb A, Trindade da Silva CA, Lee KS, Haj FG, and Hammock BD (2015) Endoplasmic reticulum stress in the peripheral nervous system is a significant driver of neuropathic pain. Proc Natl Acad Sci USA 112: 9082-9087.

Inceoglu B, Wagner K, Schebb NH, Morisseau C, Jinks SL, Ulu A, Hegedus C, Rose T, Brosnan R, and Hammock BD (2011) Analgesia mediated by soluble epoxide hydrolase inhibitors is dependent on cAMP. Proc Natl Acad Sci USA 108: 5093-5097.

Kang J, de Brito Bezerra B, Pacios S, Andriankaja O, Li Y, Tsiagbe V, Schreiner H, Fine DH, and Graves DT (2012) Aggregatibacter actinomycetemcomitans infection enhances apoptosis in vivo through a caspase-3-dependent mechanism in experimental periodontitis. Infect Immun 80:2247-2256.

Kawai T, Matsuyama T, Hosokawa Y, Makihira S, Seki M, Karimbux NY, Goncalves RB, Valverde P, Dibart S, Li YP, et al. (2006) B and T lymphocytes are the primary sources of RANKL in the bone resorptive lesion of periodontal disease. Am J Pathol 169:987-998.

Kung LH, Rajpar MH, Preziosi R, Briggs MD, and Boot-Handford RP (2015) Increased classical endoplasmic reticulum stress is sufficient to reduce chondrocyte proliferation rate in the growth plate and decrease bone growth. PLoS One 10: e0117016.

Lacey DL, Timms E, Tan HL, Kelley MJ, Dunstan CR, Burgess T, Elliott R, Colombero A, and Elliott G, Scully S, et al. (1998) Osteoprotegerin ligand is a cytokine that regulates osteoclast differentiation and activation. Cell 93: $165-176$

Levy BD, Vachier I, and Serhan CN (2012) Resolution of inflammation in asthma. Clin Chest Med 33:559-570.

Liu JY, Tsai HJ, Hwang SH, Jones PD, Morisseau C, and Hammock BD (2009) Pharmacokinetic optimization of four soluble epoxide hydrolase inhibitors for use in a murine model of inflammation. Br J Pharmacol 156:284-296.

Liu JY, Yang J, Inceoglu B, Qiu H, Ulu A, Hwang SH, Chiamvimonvat N, and Hammock BD (2010) Inhibition of soluble epoxide hydrolase enhances the anti-inflammatory effects of aspirin and 5-lipoxygenase activation protein inhibitor in a murine model. Biochem Pharmacol 79:880-887.

Marnett LJ (2009) The COXIB experience: a look in the rearview mirror. Annu Rev Pharmacol Toxicol 49:265-290.

Morisseau C, Goodrow MH, Dowdy D, Zheng J, Greene JF, Sanborn JR, and Hammock BD (1999) Potent urea and carbamate inhibitors of soluble epoxide hydrolases. Proc Natl Acad Sci USA 96:8849-8854.

Morisseau C and Hammock BD (2013) Impact of soluble epoxide hydrolase and epoxyeicosanoids on human health. Annu Rev Pharmacol Toxicol 53:37-58.

Morisseau C, Inceoglu B, Schmelzer K, Tsai HJ, Jinks SL, Hegedus CM, and Hammock BD (2010) Naturally occurring monoepoxides of eicosapentaenoic acid and docosahexaenoic acid are bioactive antihyperalgesic lipids. J Lipid Res 51: 3481-3490.

Napimoga MH, Clemente-Napimoga JT, Macedo CG, Freitas FF, Stipp RN, PinhoRibeiro FA, Casagrande R, and Verri, Jr WA (2013) Quercetin inhibits inflammatory bone resorption in a mouse periodontitis model. $J$ Nat Prod 76: 2316-2321

Napimoga MH, da Silva CA, Carregaro V, Farnesi-de-Assuncão TS, Duarte PM, de Melo NF, and Fraceto LF (2012) Exogenous administration of 15d-PGJ2-loaded nanocapsules inhibits bone resorption in a mouse periodontitis model. J Immunol 189:1043-1052

Node K, Huo Y, Ruan X, Yang B, Spiecker M, Ley K, Zeldin DC, and Liao JK (1999) Anti-inflammatory properties of cytochrome P450 epoxygenase-derived eicosanoids. Science 285:1276-1279.

Norwood S, Liao J, Hammock BD, and Yang GY (2010) Epoxyeicosatrienoic acids and soluble epoxide hydrolase: potential therapeutic targets for inflammation and its induced carcinogenesis. Am J Transl Res 2:447-457.

Okada H, Kida T, and Yamagami H (1983) Identification and distribution of immunocompetent cells in inflamed gingiva of human chronic periodontitis. Infect Immun 41:365-374.

Ortega-Gómez A, Perretti M, and Soehnlein O (2013) Resolution of inflammation: an integrated view. EMBO Mol Med 5:661-674.

Ostermann AI, Herbers J, Willenberg I, Chen R, Hwang SH, Greite R, Morisseau C, Gueler F, Hammock BD, and Schebb NH (2015) Oral treatment of rodents with soluble epoxide hydrolase inhibitor 1-(1-propanoylpiperidin-4-yl)-3-[4-(trifluoromethoxy) phenyllurea (TPPU): resulting drug levels and modulation of oxylipin pattern. Prostaglandins Other Lipid Mediat 121:131-137.

Rose TE, Morisseau C, Liu JY, Inceoglu B, Jones PD, Sanborn JR, and Hammock BD (2010) 1-Aryl-3-(1-acylpiperidin-4-yl)urea inhibitors of human and murine soluble epoxide hydrolase: structure-activity relationships, pharmacokinetics, and reduction of inflammatory pain. $J$ Med Chem 53:7067-7075. 
Schmelzer KR, Inceoglu B, Kubala L, Kim IH, Jinks SL, Eiserich JP, and Hammock BD (2006) Enhancement of antinociception by coadministration of nonsteroidal anti-inflammatory drugs and soluble epoxide hydrolase inhibitors. Proc Natl Acad Sci USA 103:13646-13651.

Schmelzer KR, Kubala L, Newman JW, Kim IH, Eiserich JP, and Hammock BD (2005) Soluble epoxide hydrolase is a therapeutic target for acute inflammation. Proc Natl Acad Sci USA 102:9772-9777.

Seo B, Coates DE, Seymour GJ, and Rich AM (2016) Unfolded protein responserelated gene regulation in inflamed periodontal tissues with and without Russell bodies. Arch Oral Biol 69:1-6.

Serhan CN, Krishnamoorthy S, Recchiuti A, and Chiang N (2011) Novel antiinflammatory-pro-resolving mediators and their receptors. Curr Top Med Chem 11:629-647.

Spector AA (2009) Arachidonic acid cytochrome P450 epoxygenase pathway. J Lipid Res 50 (Suppl):S52-S56.

Van Dyke TE (2011) Proresolving lipid mediators: potential for prevention and treatment of periodontitis. J Clin Periodontol 38 (Suppl 11):119-125.
Viswanathan S, Hammock BD, Newman JW, Meerarani P, Toborek M, and Hennig B (2003) Involvement of CYP 2C9 in mediating the proinflammatory effects of linoleic acid in vascular endothelial cells. J Am Coll Nutr 22:502-510.

Williams JM, Murphy S, Burke M, and Roman RJ (2010) 20-hydroxyeicosatetraeonic acid: a new target for the treatment of hypertension. J Cardiovasc Pharmacol 56:336-344. Yamada H, Nakajima T, Domon H, Honda T, and Yamazaki K (2015) Endoplasmic reticulum stress response and bone loss in experimental periodontitis in mice. $J$ Periodontal Res 50:500-508.

Address correspondence to: Dr. Bruce D. Hammock, Department of Entomology and Nematology and University of California, Davis Comprehensive Cancer Center, National Institute on Environmental Health Sciences-University of California, Davis Superfund Research Program PI, National Institutes of Health Biotechnology Training Program, University of California, Davis, One Shields Avenue, Davis, CA 95616. E-mail: fax bdhammock@ucdavis.edu 